Published in final edited form as:

Shchukina, E., Shchukin, D., Grigoriev, D. (2016). Halloysites and mesoporous silica as inhibitor nanocontainers for feedback active powder coatings. Progress in Organic Coatings X(XX), XX-XX, doi:10.1016/j.porgcoat.2015.12.013

Available online since 11 January 2016

\title{
Halloysites and mesoporous silica as inhibitor nanocontainers for feedback active powder coatings
}

\begin{abstract}
:
We carried out comparative study of the effect of inhibitor-loaded nanocontainers on corrosion protection performance of polyepoxy powder coatings employing neutral salt-spray test $(5 \% \mathrm{NaCl}, 35 \circ$, different time). We demonstrated that halloysites and mesoporous silica particles loaded with corrosion inhibitor 8-hydroxyquinoline can be homogeneously distributed in powder coating effectively reducing corrosion of the metal substrate over $1000 \mathrm{~h}$ of salt-spray test. Addition of only $2 \mathrm{wt}$.\% of inhibitor encapsulated in both nanocontainers to the powder coating is sufficient to decrease the delamination effect by 2-3 times and suppress the formation of the pitting corrosion.
\end{abstract}

\section{Keywords:}

Nanocontainer

Self-healing

Halloysite

Mesoporous silica

Salt spray test

Anticorrosion 


\title{
Halloysites and mesoporous silica as inhibitor nanocontainers for feedback active powder coatings
}

\author{
Elena Shchukina $^{a}$, Dmitry Shchukin ${ }^{a, *}$, Dmitry Grigoriev ${ }^{b}$ \\ a Stephenson Institute for Renewable Energy, University of Liverpool, Liverpool L69 7ZF, UK \\ b Max Planck Institute of Colloids and Interfaces, Golm D14476 Germany
}

*Corresponding author. Tel.: +0044151952304. E-mail address: d.shchukin@liverpool.ac.uk

(D. Shchukin).

\section{Introduction}

Self-healing activity of the materials is based on their feedback action. Causes-effect relations between material constituents form a loop where the output responding to the environment input (like local $\mathrm{pH}$-changes during corrosion process) [1]. The input can be also an external signal applied to the material (light or mechanical force). The output is the restoring functionality of the initial material, in our case corrosion protection ability of the coatings. First simple approach for providing feedback healing to the organic coatings is to directly introduce corrosion inhibitors in the pretreatment, primer or topcoat layers of the coatings [2]. The idea behind is the response to the coating damage by diffusive or stimuliinduced release of the inhibitor from the coating matrix. Contrary to the expectations, direct introduction of the inhibitor into coating matrix results, in most cases, in the significant reduction of its corrosion protection performance [3]. Very low solubility of inhibitor leads to its deficit in the damaged area. In the opposite case of too high solubility, metal substrate can be protected for only a relatively short time due to rapid leaching of inhibitor from the coating. Inhibitor freely dispersed inside the active matrix is often subjected to spontaneous leakage [4]. Another drawback, which can appear due to high solubility, is the osmotic pressure initiating blistering and, finally, delamination of the coating $[5,6]$.
Very successful approach to impart feedback functionality to a coating is incorporation into coating matrix the encapsulated inhibitor [5,7]. Capsules or nanocontainers can isolate encapsulated corrosion inhibitor from coating matrix, terminate spontaneous leakage of inhibitor and, at the same time, provide controlled release of the inhibitor directly into the corroded area on demand. In general, containers in the size range of $20 \mathrm{~nm}$ to $50 \mathrm{~J} . \mathrm{Lm}$ require shell which has to be stable, permeable to release/upload inhibitors and possesses other desired functionalities (magnetic, catalytic, conductive, targeting, etc.). There are several approaches demonstrated so far for nanocontainer systems: (i) polymer containers [8], (ii) halloysites [9], (iii) nanocontainers with polyelectrolyte shell [10], (iv) layered double hydroxides [11], (v) ion-exchange organic resins [12], (vi) conductive polymer matrixes [13] and, finally, (vii) mesoporous inorganic materials [14].

The current level of the development of nanocontainer-based self-healing coatings has large number of the highly-efficient examples on the laboratory scale $[15,16]$. So, this research can be checked for feasibility of commercial application. However, there are two main difficulties to make this transition: the costs of the nanocontainers and the availability of the valid results of the industrial corrosion protection tests (mostly salt-spray tests). The first problem requires the search of the cheap nanocontainer hosts which can be available in large-scale quantities. Halloysites and mesoporous silica particles can be perfect candidates. They are much cheaper comparing to the other types of nanocontainers and commercially available in large quantities [17]. Moreover, the interest in using inorganic nanocontainers is that their mechanical and 
thermal stability allow their utilisation in different coating layers (pre-treatment, primer, topcoat) subjected to high mechanical loads or significant thermal stresses.

Halloysites are defined as two-layered natural aluminosilicates, chemically similar to kaolin, which have a predominantly hollow tubular structure in the submicrometer range [18]. Self-healing properties of the benzotriazole and 8-hydroxyquinoline loaded halloysite nanotubes were studied in zirconia-silica sol-gel coatings deposited on the surface of aluminium alloy A2024 by periodic measurements of SVET (Scanning Vibrating Electrode technique) current density profiles [9]. The maximal anodic current reduced down to the noise level within $4.5 \mathrm{~h}$ for benzotriazole-loaded halloysites while the maximal current density did not remain constant at the noise level for 8-hydroxyquinoline-loaded halloysites. To prevent undesirable leakage of the loaded inhibitor from the halloysite interior at neutral $\mathrm{pH}$, the outer surface of the inhibitorloaded halloysite nanotubes was modified by deposition of alternating polyelectrolyte multilayers (poly(allylamine hydrochloride)/poly(styrene sulfonate)) [19]. Loading benzotriazole, mercaptobenzimidazole and mercaptobenzothiazole into halloysites made them active for protection of copper $[20,21]$. The release rate of inhibitor was controlled by the formation of metal-benzotriazole stoppers at tube endings. Formation of the $\mathrm{pH}$-controlled metal-inhibitor complex was studied for $\mathrm{Cu}(\mathrm{II}), \mathrm{Fe}(\mathrm{II}), \mathrm{Fe}(\mathrm{III})$ and Co(II) ions. The best release control was achieved for $\mathrm{Cu}$-complex. Some corrosion was evident within the first fifteen days, but then it was suppressed with the release of inhibitors in the coating defects. Efficiency of the halloysite lumen loading ascended in the order of benzotriazole $<$ mercaptobenzothiazole $<$ mercaptobenzimidazole corrosion inhibitors.

Another type of the nanocontainers with inorganic scaffold is mesoporous particles. The material of these particles has to be inert to the corrosion inhibitors. Therefore, only titania and silica particles can be applied for most inhibitors, and the silica ones have beneficial properties-high specific surface area and inertness to the ultraviolet irradiation, which is important for outdoor coatings. Due to these characteristics, mesoporous silica particles were quickly developed as delivery tool for biomedical applications $[22,23]$. The incorporation of mesoporous nanocontainers $\left(\approx 1000 \mathrm{~m}^{2} / \mathrm{g}\right.$ specific surface area) into inorganic sol-gel coatings improved significantly the coating corrosion resistance [24]. On one hand, the coating barrier properties were enhanced by reinforcement of the coating matrix due to introduction of mechanically stable, robust silica nanoparticles. On the other hand, the large amount of encapsulated inhibitor (up to $80 \mathrm{wt} . \%$ ) and its controlled release upon corrosion attack provided superior corrosion inhibition. Additional advantage of the silica nanocontainers is the possibility to tailor hydrophobic surface functionality to disperse them in solvent-born coatings. Mesoporous $\mathrm{SiO}_{2}$ functionalised with octyl groups and loaded with benzotriazole showed tenfold greater corrosion protection performance in polyester-based commercial coatings than that coating without nanocontainers [25]. Silica nanocontainers with $80 \mathrm{~nm}$ size demonstrated high barrier (Electrochemical Impedance Spectroscopy, EIS) and selfhealing (SVET) properties of the coatings while the increase of the nanocontainer size to $700 \mathrm{~nm}$ reduced the coating corrosion resistance by the factor of two remaining coating physical properties (thickness and adhesion) comparable to $80 \mathrm{~nm}$ nanocontainers [26]. Similar to the halloysite nanotubes, the release of the encapsulated inhibitor can be controlled by metal complexes formed in the pore openings on the silica surface. $\mathrm{Cu}$-benzotriazole complex formed at the openings of silica mesopores can release the encapsulated inhibitor and biocide (benzalkonium chloride) either at $\mathrm{pH}$ lower than 5 or in presence of sulphide ions (about $0.6 \mathrm{ppm}$ ) providing corrosion protection and antifouling action at the same time [27]. Immobilisation of photoresponsive azobenzene molecular switches into the npores instead of metal ions makes mesoporous silica nanocontainers sensitive to UV light reversibly releasing/uptaking benzotriazole under UV-vis irradiation and thus showing continuous self-healing ability under external stimuli [28].

Despite large number of the papers devoted to the nanocontainer-based self-healing coatings, most of them use lab-scale analytical methods for characterisation of their selfhealing performance: EIS, polarisation, SVET and various adapted electrochemical techniques. Only a few papers [29] analysed the efficiency of the nanocontainer-based coatings using industrial methods. Here, we attempt to reduce this "transfer gap" and present comparative analysis, done by industrial neutral saltspray test (ISO 9227), of the corrosion protection performance of halloysites and mesoporous silica particles as nanocontainers loaded with corrosion inhibitor 8-hydroxyquinoline (8-HQ) and impregnated into polyester powder coating. Coatings with and without nanocontainers were tested on bare low carbon steel substrates.

\section{Experimental procedure}

\subsection{Materials}

Corrosion inhibitor 8-hydroxyquinoline, ethanol, acetone, $\mathrm{HCl}$, $\mathrm{NaOH}$ and $\mathrm{NaCl}$ were purchased from Sigma-Aldrich and used without further purification. Halloysites were provided by Atlas Mining Company (Dragon mine deposit, Utah, USA) and mesoporous silica particles were purchased from Grace, USA (SYLOID ${ }^{\circledR}$ C803 silica). Halloysites are naturally occurring layered kaolin aluminosilicates with hollow tubular structure. The aluminum hydroxide and the silicon oxide layers are bond covalently with each other. The bilayer rolls up to a tube, i.e. a hollow cylinder with alumina layer inside and silica layer outside [30].

Halloysite nanotubes from Dragon Mine deposit have elongated form with average length around $1 \mathrm{~J} . \mathrm{Lm}$ with outer diameter around $50 \mathrm{~nm}$ and inner lumen of $15-20 \mathrm{~nm}$ diameter (Fig. 1A).
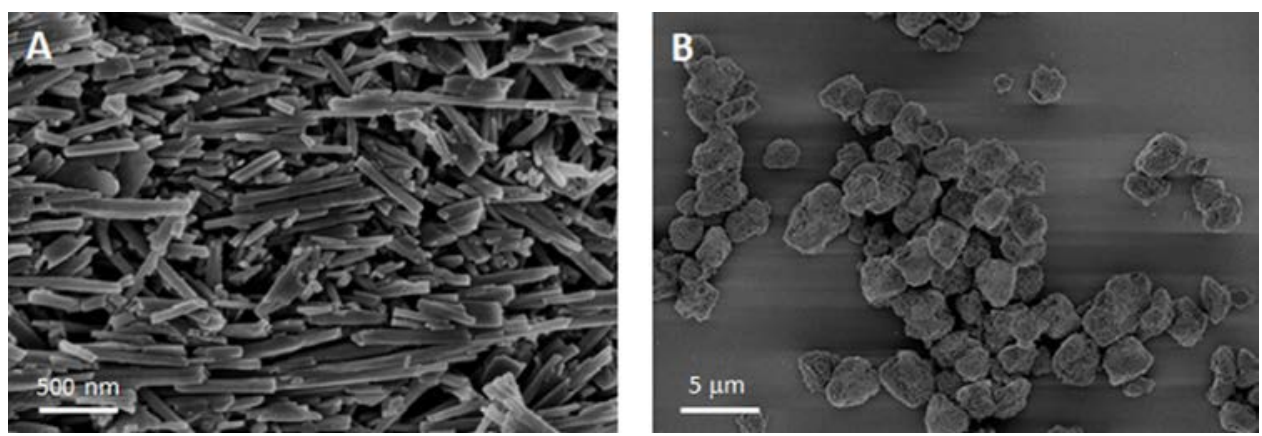

Fig. 1. SEM images of halloysite nanotubes and mesoporous $\mathrm{SiO}_{2}$ particles used as nanocontainers. 
Mesoporous SYLOID ${ }^{\circledR}$ C803 silica particles have irregular shape and average size of 3-4 J.Lm (Fig. 1B). BET analysis demonstrated specific surface area of $60 \mathrm{~m}^{2} / \mathrm{g}$ with pore volume of $0.2 \mathrm{~cm}^{3} / \mathrm{g}$ for halloysites and $285 \mathrm{~m}^{2} / \mathrm{g}$ with pore volume of $1.4 \mathrm{~cm}^{3} / \mathrm{g}$ for mesoporous $\mathrm{SiO}_{2}$.

Powder coatings were prepared on the basis of polyester resin Crylcoat 2698-3 provided by Cytec Industries, Inc., USA. Cold rolled low carbon steel plates for neutral salt spray tests were purchased from Rocholl GmbH (Germany) and had dimensions $\mathrm{L} \times \mathrm{W} \times \mathrm{T}=150 \times 75 \times 2 \mathrm{~mm}$ and surface cleanliness grade SA2.5.

\subsection{Preparation of the coatings}

Loading of the nanocontainers with 8-hydroxyquinoline was performed from 33 wt.\% acetone solution under reduced pressure. $50 \mathrm{ml}$ of 33 wt.\% 8-HQ were mixed with $5 \mathrm{~g}$ of empty nanocontainers (halloysites or mesoporous silica particles) and then introduced into a desiccator with a reduced pressure. The air goes out from the nanocontainer inner volume being replaced by the solution containing corrosion inhibitor. The loading was performed three times followed by centrifugation in water at $5000 \mathrm{rpm}$ each time. The maximum 8-HQ loading inside halloysite nanotubes is $20 \mathrm{wt} . \%$ while for $\mathrm{SiO}_{2}$ nanoparticles loading capacity reached 77 wt.\%.

On the next stage, 8-HQ loaded nanocontainers (10 wt.\% of halloysites or 2.6 wt.\% of $\mathrm{SiO}_{2}$ ) were mixed with the powder coating composition using a laboratory extruder in order to achieve the same concentration of inhibitor in the final coating for both halloysites and mesoporous silica-2 wt.\% of inhibitor in each coating. The coating was deposited on the metal substrate and cured for 10 min at $190{ }^{\circ} \mathrm{C}$ object temperature. The final coating thickness was in the range $85 \pm 5 \mathrm{~J} . \mathrm{Lm}$ for all samples.

\subsection{Characterisation}

Scanning electron microscopy, SEM (Zeiss Gemini LEO 1550) was employed to analyse the structure of nanocontainers. For characterisation of particles, a droplet of the particle water suspension was placed on the sample holder and left to dry at atmospheric pressure and room temperature. All samples were sputtered with gold/palladium mixture to avoid electron charging of the samples during SEM analysis. The samples were studied using an operating voltage of $3 \mathrm{kV}$ and different magnifications. Nanocontainers in the coatings were analysed by the cross-section of coated steel plates with the following embedding of cut out part in the epoxy resin matrix with subsequent careful polishing the cross-section facet. The coating thickness was measured with a coating thickness gauge, Surfix ${ }^{\circledR}$ Pro S, from PHYNIX, Germany.
Anticorrosion performance was tested by neutral salt-spray test (Ascott CC450XP salt spray chamber, ISO 9227, 5 wt.\% NaCl, $35{ }^{\circ} \mathrm{C}$, $1 \mathrm{~mm}$ scribe, $260 \mathrm{~h}$ and $1000 \mathrm{~h}$ time). In order to perform the numerical evaluation of delamination extent for the coated steel plates after $1000 \mathrm{~h}$ of test duration, the area around the scribe, where the delaminated coating was detached, was stained by the special black dye. This procedure allowed the strong enhancement of the contrast between the bare metal surface partially coloured by the corrosion products and the intact coating surrounding delaminated area. The photographs of stained plates were digitised and transformed to the binary form. The total amount of black pixels was counted and divided by the length of the scribe. Finally, the backward transformation yielded the averaged value of the delamination independent of the specific measuring point across the scribe. Release studies of 8-HQ at different $\mathrm{pH}$ were performed spectroscopically adjusting the $\mathrm{pH}$ of the solution by $\mathrm{HCl}$ or $\mathrm{NaOH}$. Thermogravimetric analysis (TGA) was carried out using a TG 209 F1 device (Netzsch-Gerätebau GmbH, Germany) with a heating rate $10 \mathrm{~K} / \mathrm{min}$ under $\mathrm{N}_{2}$ atmosphere. The specific surface area and pore volume of nanocontainers were obtained by means of $\mathrm{N}_{2}$ adsorption/desorption measurements (QuadraSorb SI Surface Area Analyzer and Pore Size Analyzer, Quantachrome Instruments, USA).

\section{Results and discussion}

The highest loading efficiency has been observed for both nanocontainer types after third loading cycle (see Section 2). First loading cycle provided 12 wt.\% inhibitor loading for halloysites and 34 wt.\% of loaded inhibitor for mesoporous $\mathrm{SiO}_{2}$, as was shown by TGA analysis. Next two cycles lead to the loading limit of $20 \mathrm{wt} . \%$ of 8-HQ for halloysites and 77 wt.\% for silica. Further repetition of the loading did not result the increase of the quantity of 8-HQ in the nanocontainers clearly indicating loading limit achieved after third loading cycle.

Complete release of the encapsulated 8-HQ appeared after $5 \mathrm{~h}$ in water media at $\mathrm{pH}=7$ for both halloysites and silica particles (Fig. 2A). Mesoporous silica shows higher release rate at initial stages due to the network of open pores while halloysite nanotubes have two "exits" and, therefore, longer time is needed for 8-HQ to diffuse from the inner halloysite lumen. The release speed can be significantly accelerated in acidic or alkaline $\mathrm{pH}$ range because of the amphoteric structure of the corrosion inhibitor (Fig. 2B). Formation of the ionic forms of 8-HQ at low (cationic) and high (anionic) $\mathrm{pH}$ values initiates 100-times increase of the release speed which can be effectively employed for smart response of the 8-HQ loaded nanocontainers to the corrosion start. The chosen acidic $(<\mathrm{pKa} 1)$ and basic $(>\mathrm{pKa} 2) \mathrm{pH}$ values are typically achieved
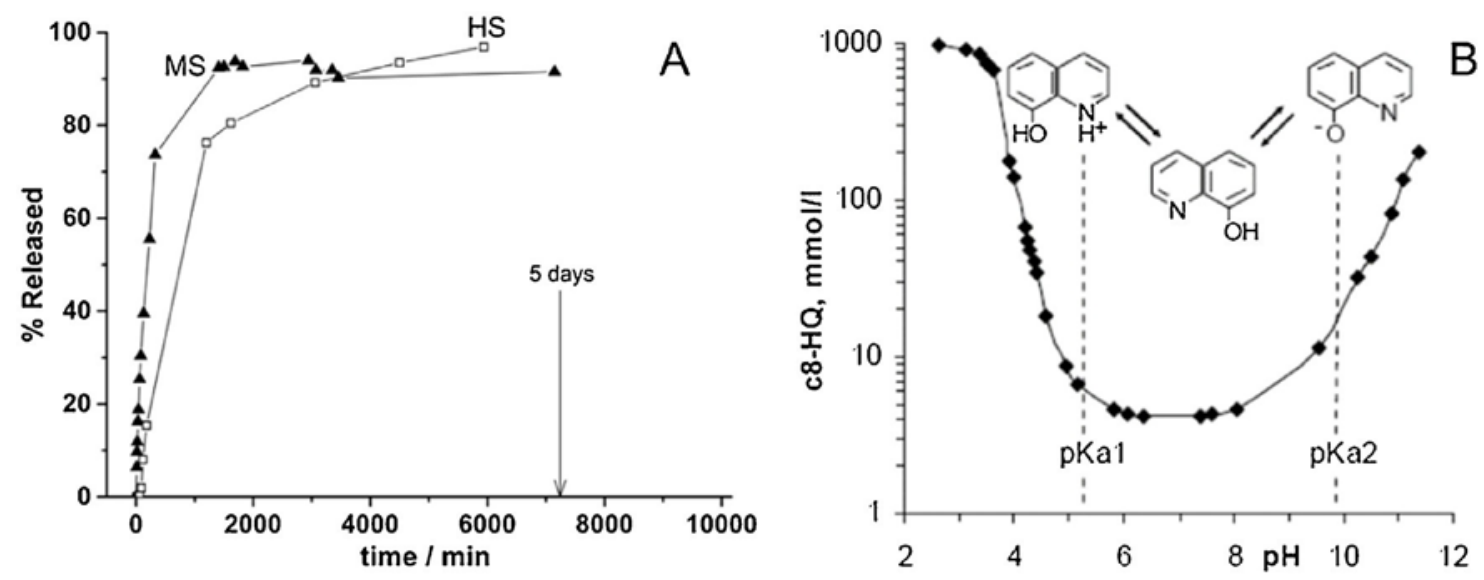

Fig. 2. Release of the encapsulated 8-HQ at $\mathrm{pH} 7$ in water for both nanocontainers (A) and $\mathrm{pH}$ accelerated solubility of 8-HQ (B). 

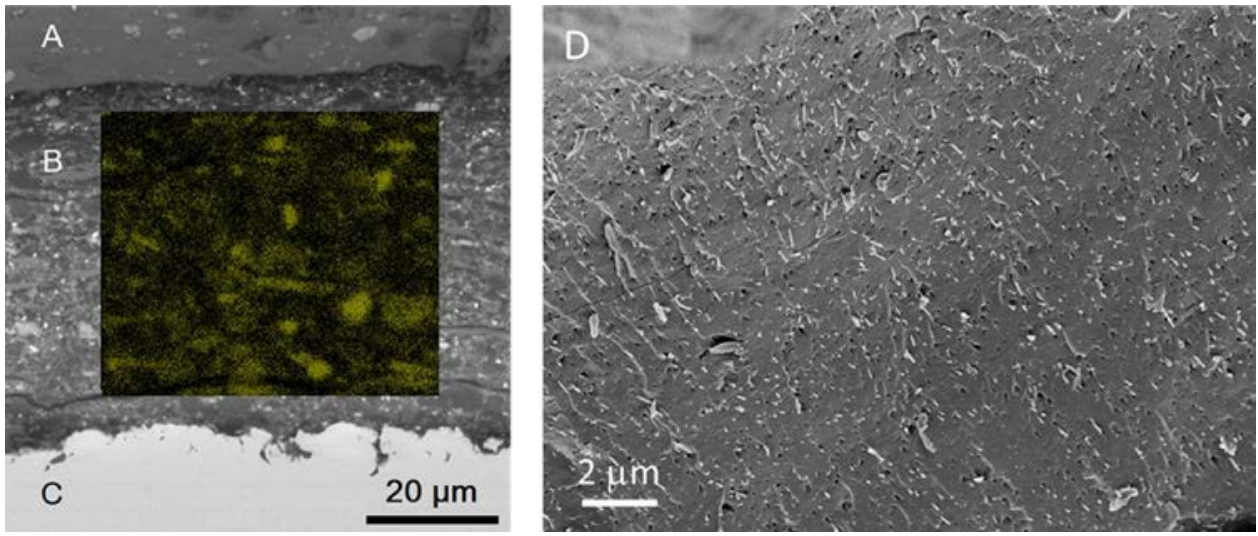

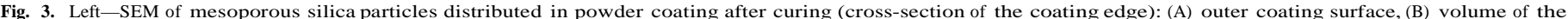

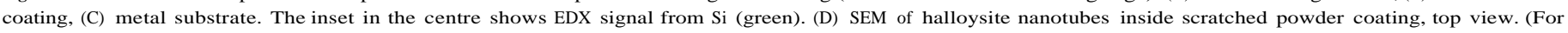
interpretation of the references to colour in this figure legend, the reader is referred to the web version of this article.)

during the corrosion of steel or aluminium alloys and can be utilised as triggers [5]. On the other hand, the reduced release at $\mathrm{pH}=7$ should limit the leaching of inhibitor from the coating. The release studies revealed a $\mathrm{pH}$-responsive 8-HQ release from both types of nanocontainers.

The nanocontainers are well dispersed in the coating. Fig. 3 shows that both nanocontainers do not create aggregates in the coating preventing the loss of the coating integrity. Even several aggregates can cause severe damage to the coating barrier properties [11]. Nanocontainers are distributed homogeneously in the whole coating volume with halloysites retaining their elongated tubular structure after coating deposition and curing. Average concentration of halloysites in the coating, as calculated from SEM/EDX data, is around 15 per $1 \mathrm{J.m}^{3}$. Bigger $\mathrm{SiO}_{2}$ particles are less concentrated with approximately 5 particles per $1 \mathrm{~J} . \mathrm{Lm}^{3}$. The concentration of nanocontainers is in both cases enough to provide sufficient release and adsorption of the inhibitor in damaged areas of the coating since two-three inhibitor layers are enough to terminate corrosion propagation [31].
Previously, we used SVET and EIS for analysis of the selfhealing and barrier properties of inhibitor-loaded nanocontainers in coatings $[9,19]$. Mentioned laboratory tests clearly demonstrated the efficiency of the inhibitor-loaded halloysite nanotubes and mesoporous particles on laboratory level. However, more rigorous industrial tests are necessary before commercial application of the nanocontainer-based self-healing coatings.

As one can see in Fig. 4, pure commercial coating reveals corrosion and degradation during first $260 \mathrm{~h}$ of the neutral salt spray test. There is corrosion propagation under the coating from artificial scribe and also signs of the blistering corrosion. Coatings with both inhibitor-loaded nanocontainers show much better corrosion protection ability with small signs of corrosion propagation under artificial scratch and no blistering effects.

At the same time, direct addition of the 8-HQ in the concentration even 2.5 times higher (5 wt.\%) than for encapsulated 8-HQ (2 wt.\%) demonstrated complete deterioration of the coating with many signs of pitting corrosion (Fig. 5). The latter indicates reduction of the barrier properties of the coating due to the interaction
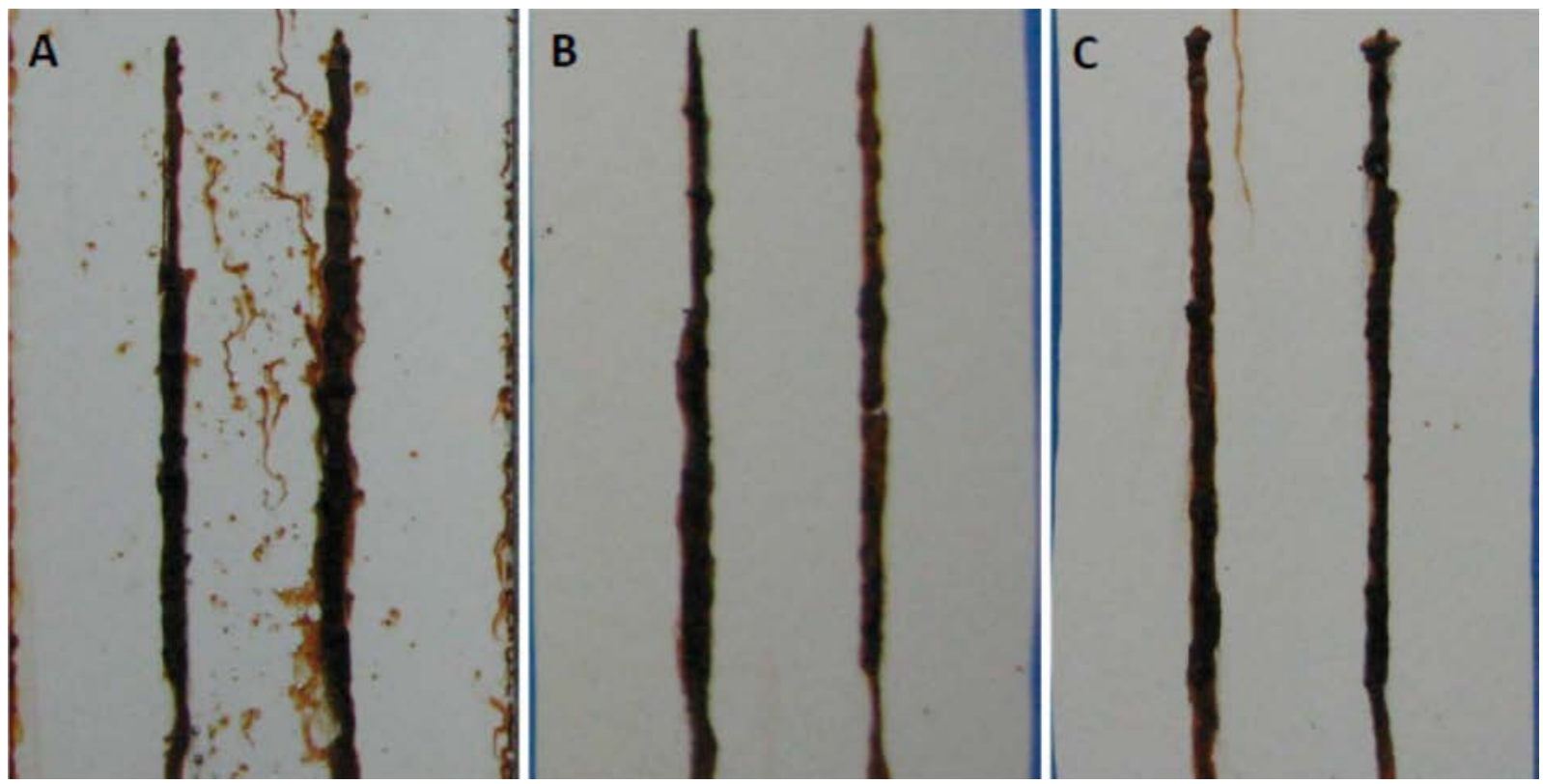

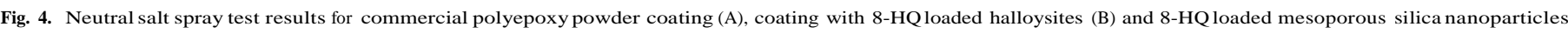
(C) after $260 \mathrm{~h}$ of the test. 


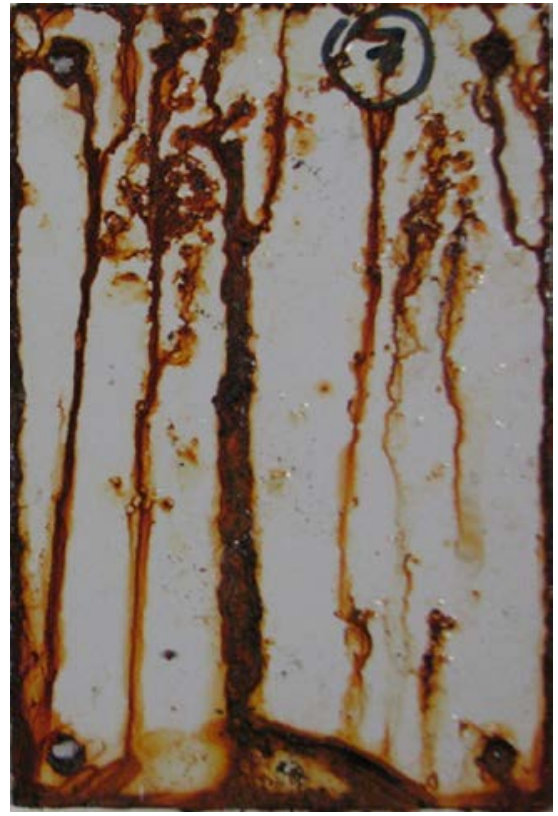

Fig. 5. Neutral salt spray test results for commercial polyepoxy powder coating with $5 \mathrm{wt} . \%$ of directly added 8-hydroxyquinoline after $260 \mathrm{~h}$ of the test.

of the hydroxyl groups of 8-HQ with epoxy derivatives of the coating matrix, which forms micropores in the coating facilitating the penetration pathways for $\mathrm{Cl}^{-}$ions.

Continuous reduction of the corrosion protection of the pure commercial coating with many pits and delamination in the scratched area leading to the total degradation of the coating were demonstrated after $1000 \mathrm{~h}$ of the test while the coatings with 8-HQ loaded nanocontainers are still stable (Fig. 6). Quantification of salt spray test showed $>5 \mathrm{~mm}$ delamination for pure commercial polyepoxy coating after $1000 \mathrm{~h}$. Introducing 8-HQ loaded halloysites or mesoporous silica particles reduced the delamination to $1-2 \mathrm{~mm}$ and only small number of pitting corrosion sites were found for coating with mesoporous silica particles.
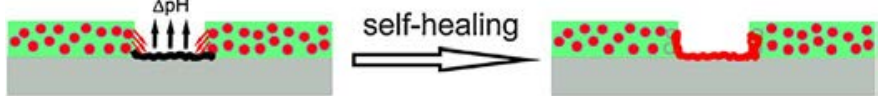

Fig. 7. Schematic presentation of the corrosion protection behaviour of the polyepoxy powder coatings with 8-hydroxyquinoline loaded nanocontainers.

\section{Conclusion}

Summarising the data presented above, we can conclude that the effect of the inhibitor-loaded nanocontainers is based on the $\mathrm{pH}$-controlled controlled release of the 8-hydroxyquinolile inside the damaged (or corroded) area of the polyepoxy powder coatings. Local decrease or increase of the $\mathrm{pH}$ due to the corrosion process immediately accelerates the solubility of the encapsulated 8-hydroxyquinoline provoking its diffusion from nanocontainers neighbouring to the defected area where it then chemisorbs on the anodic corrosion sites and terminates corrosion (Fig. 7) [32].

Neutral salt spray tests (Ascott CC450XP salt spray chamber, ISO 9227, 5 wt.\% $\mathrm{NaCl}, 35^{\circ} \mathrm{C}, 1 \mathrm{~mm}$ scribe, $260 \mathrm{~h}$ and $1000 \mathrm{~h}$ time) demonstrated different corrosion protection performance of pure commercial polyepoxy powder coating, powder coating with directly incorporated 8-hydroxyquinoline (5 wt.\%) and power coatings with 8-hydroxyquinoline loaded halloysites or mesoporous silica particles. Addition of only $2 \mathrm{wt} \%$ of inhibitor encapsulated in both nanocontainers is sufficient to decrease the delamination effect by 2-3 times and suppress the formation of the pitting corrosion after $1000 \mathrm{~h}$ of salt spray test as compared to the pure commercial coating. At the same time, direct addition of 8-Hydroxyquinoline into the coating resulted in complete coating degradation even after $260 \mathrm{~h}$ of the salt spray test. This indicates that (i) the encapsulated inhibitor can be effectively used for corrosion protection due to its isolation from the coating matrix inside cheap and abundant halloysite or silica nanocontainers where it stays in active, not-bounded form and (ii) halloysites and mesoporous silica particles can be homogeneously distributed in powder coating without formation of the aggregates.
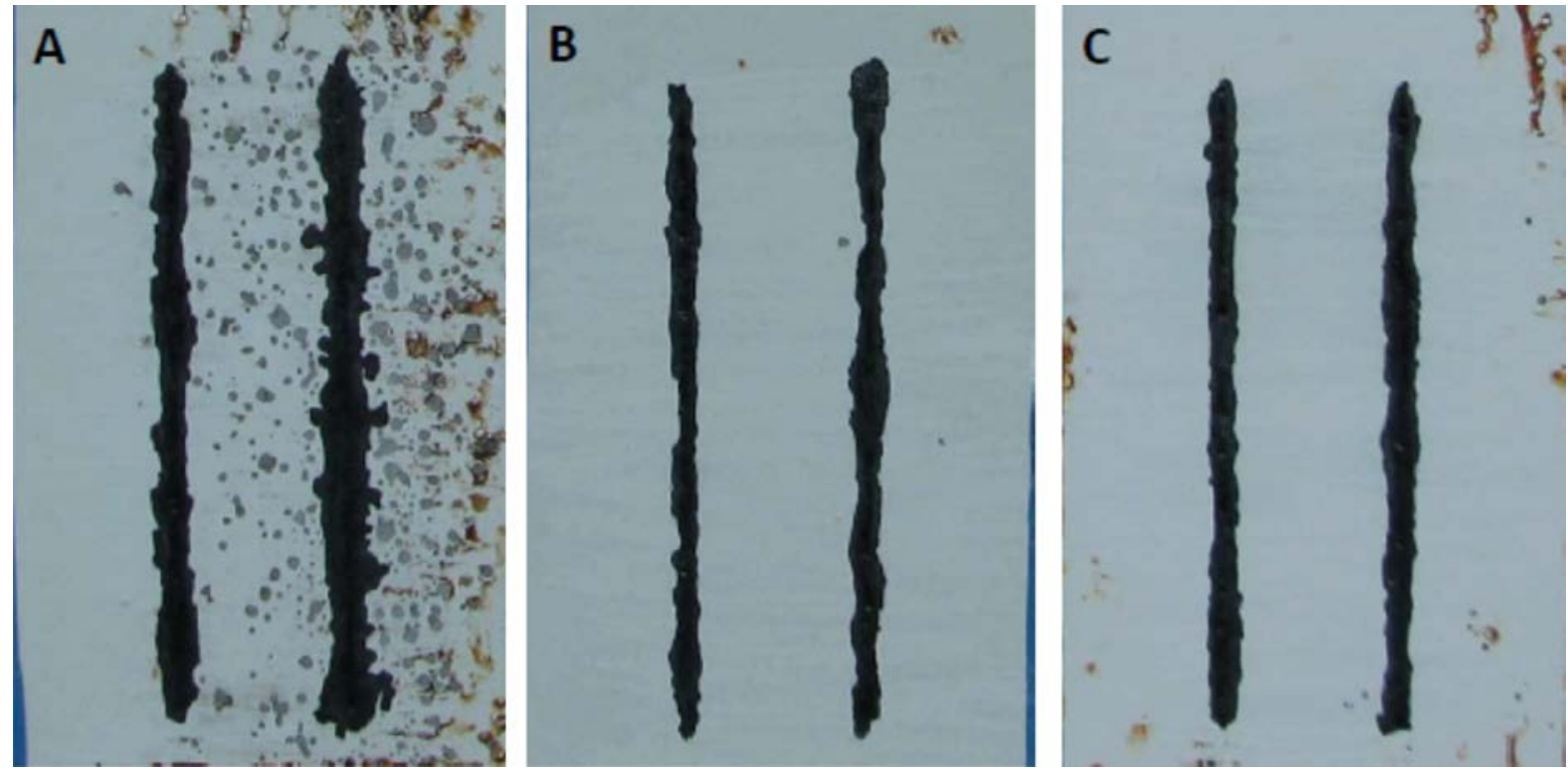

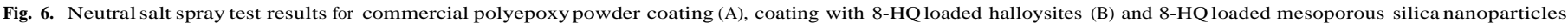
(C) after $1000 \mathrm{~h}$ of the test. 


\section{Acknowledgements}

DS acknowledges ERC 647969 Consolidator grant ENERCAPSULE for financial support.

DG is grateful for the financial support of the Federal Ministry of Economics and Energy (BMWi), Germany 03EFCBB028 in the framework of "EXIST Transfer of Research" program (Project "SigmA" Nr. 03EFCBB028) and G8 project "SMARTCOAT", G8MUREFU2-FP-377-008. We thank Dr. M. Schenderlein and Mr. E. Scherke for the help in experimental work.

\section{References}

[1] J.J. DiStefano, A.R. Stubberud, I.J. Williams, Schaum's Outline of Theory and Problems of Feedback and Control Systems: Continuous (Analog) and Discrete (Digital), McGraw-Hill, New York, NY, 1995.

[2] E.W. Brooman, Met. Finish. 100 (2002) 42-53.

[3] M. Garcia-Heras, A. Jimenez-Morales, B. Casal, J.C. Galvan, S. Radzki, M.A. Villegas, J. Alloys Compd. 380 (2004) 219-224.

[4] A.J. Vreugdenhi, M.E. Woods, Prog. Org. Coat. 53 (2005) 119-125.

[5] D.G. Shchukin, H. Mohwald, Small 3 (2007) 926-943.

[6] D. Raps, T. Hack, J. Wehr, M.L. Zheludkevich, A.C. Bastos, M.G.S. Ferreira, O. Nuyken, Corros. Sci. 51 (2009) 1012-1021.

[7] D.G. Shchukin, H. Mohwald, Chem. Commun. 47 (2011) 8730-8739.

[8] S.R. White, N.R. Sottos, P.H. Geubelle, J.S. Moore, M.R. Kessler, S.R. Sriram, E.N. Brown, S. Viswanathan, Nature 409 (2001) 794-797.

[9] D. Fix, D.V. Andreeva, D.G. Shchukin, Y.M. Lvov, H. Möhwald, Adv. Funct. Mater. 19 (2009) 1720-1727.

[10] A.M. Pavlov, S.A. Gabriel, G.B. Sukhorukov, D.J. Gould, Nanoscale 7 (2015) 9686-9693.

[11] J. Tedim, M.L. Zheludkevich, A.C. Bastos, A.N. Salak, A.D. Lisenkov, M.G.S. Ferreira, Electrochim. Acta 117 (2014) 164-171.

[12] G. Williams, S. Geary, H.N. McMurray, Corros. Sci. 57 (2012) 139-147.
[13] B.D. Mert, B. Yazici, S.B. Lyon, Corros. Eng. Sci. Technol. 48 (2013) 506-512.

[14] D. Borisova, M. Schenderlein, D.G. Shchukin, ACS Appl. Mater. Interfaces 4 (2012) 2931-2939.

[15] H. Wei, Y. Wang, J. Guo, N.Z. Shen, D. Jiang, X. Zhang, X. Yan, J. Zhu, Q. Wang, L. Shao, H. Lin, S. Wei, Z. Guo, J. Mater. Chem., A 3 (2015) 469-480.

[16] M.L. Zheludkevich, J. Tedim, M.G.S. Ferreira, Electrochim. Acta 82 (2012) 314-323.

[17] E. Joussein, S. Petit, J. Churchman, B. Theng, D. Righi, B. Delvaux, Clay Miner. 40 (1992) 383-426.

[18] Y.M. Lvov, D.G. Shchukin, H. Mohwald, R.R. Price, ACS Nano 2 (2008) 814-820

[19] D.G. Shchukin, S.V. Lamaka, K.A. Yasakau, M.L. Zheludkevich, M.G.S. Ferreira, H. Mohwald, J. Phys. Chem., C 112 (2008) 958-964.

[20] E. Abdullayev, R. Price, D. Shchukin, Y. Lvov, Appl. Mater. Interfaces 1 (2009) 1437-1443.

[21] E. Abdullayev, V. Abbasov, A. Tursunbayeva, V. Portnov, H. Ibrahimov, G. Mukhtarova, Y. Lvov, Appl. Mater. Interfaces 5 (2013) 4464-4471.

[22] A. López-Noriega, D. Arcos, M. Vallet-Regí, Chem. Eur. J. 16 (2010) 10879-11886.

[23] V. Cauda, A. Schlossbauer, J. Kecht, A. Zurner, T. Bein, J. Am. Chem. Soc. 131 (2009) 11361-11370.

[24] D. Borisova, H. Möhwald, D.G. Shchukin, ACS Nano 5 (2011) 1939-1946.

[25] M.J. Hollamby, D. Fix, I. Dönch, D. Borisova, H. Möhwald, D. Shchukin, Adv. Mater. 23 (2011) 1361-1365.

[26] D. Borisova, D. Akcakayıran, M. Schenderlein, H. Möhwald, D. Shchukin, Adv. Funct. Mater. 23 (2013) 3799-3812.

[27] Z. Zheng, X. Huang, M. Schenderlein, D. Borisova, R. Cao, H. Möhwald, D. Shchukin, Adv. Funct. Mater. 23 (2013) 3307-3314.

[28] T. Chen, R. Chen, Z. Jin, J. Liu, J. Mater. Chem., A 3 (2015) 9510-9516.

[29] M.L. Zheludkevich, S.K. Poznyak, L.M. Rodrigues, D. Raps, T. Hack, L.F. Dick, T. Nunes, M.G.S. Ferreira, Corros. Sci. 52 (2010) 602-611.

[30] E. Ruiz-Hitzky, K. Ariga, Y.M. Lvov, Bio-inorganic Hybrid Nanomaterials: Strategies, Syntheses, Characterization and Applications, Wiley-VCH Verlag GmbH \& Co. KGaA, Weinheim, Germany, 2008.

[31] D. Grigoriev, D. Akcakayiran, M. Schenderlein, D. Shchukin, Corrosion 70 (2014) 446-463.

[32] V.S. Sastri, Corrosion Inhibitors: Principles and Applications, John Wiley and Sons, New York, 1998, ISBN-13: 978-0471976080. 